\title{
Reflexive and Voluntary Orienting of Visual Attention: Time Course of Activation and Resistance to Interruption
}

\author{
Hermann J. Müller \\ Department of Psychology, Birkbeck College \\ University of London, England
}

\author{
Patrick M. A. Rabbitt \\ Age and Cognitive Performance Research Centre \\ University of Manchester, England
}

\begin{abstract}
To study the mechanisms underlying covert orienting of attention in visual space, subjects were given advance cues indicating the probable locations of targets that they had to discriminate and localize. Direct peripheral cues (brightening of one of four boxes in peripheral vision) and symbolic central cues (an arrow at the fixation point indicating a probable peripheral box) were compared. Peripheral and central cues are believed to activate different reflexive and voluntary modes of orienting (Jonides, 1981; Posner, 1980). Experiment 1 showed that the time courses of facilitation and inhibition from peripheral and central cues were characteristic and different. Experiment 2 showed that voluntary orienting in response to symbolic central cues is interrupted by reflexive orienting to random peripheral flashes. Experiment 3 showed that irrelevant peripheral flashes also compete with relevant peripheral cues. The amount of interference varied systematically with the interval between the onset of the relevant cue and of the distracting flash (cue-flash onset asynchrony) and with the cuing condition. Taken together, these effects support a model for spatial attention with distinct but interacting reflexive and voluntary orienting mechanisms.
\end{abstract}

In daily life persons move their eyes so as to foveate parts of the visual field to which they wish to attend. However, it is well established that even without making eye movements, persons can choose to attend to input at particular extrafoveal locations (e.g., Colegate, Hoffman, \& Eriksen, 1973; Eriksen \& Colegate, 1971; Eriksen \& Hoffman, 1972; Posner, 1980; Posner, Snyder, \& Davidson, 1980). Posner has termed this covert visual orienting.

Posner (1980) proposed that there are two modes of control over covert visual orienting: The first mode is exogenous, involving reflexive orienting in response to salient stimuli in the visual field, such as a peripheral flash (peripheral cue). The second mode is endogenous, involving voluntary orienting in response to symbolic indicators, such as a central arrow (central cue). Posner's two modes of control over spatial attention are a distinction between two ways in which one common orienting mechanism, which brings limited-capacity attention processes to bear on stimuli at selected spatial regions, can be activated.' Henceforth these two modes of orienting are referred to as reflexive and voluntary (in analogy with the guidance of saccadic eye movements).

Jonides (1981) extended this distinction by proposing that reflexive and voluntary orienting differ in their automaticity.

This research was supported by Medical Research Council grant G84/03193N to Patrick M. A. Rabbitt and by Deutsche Forschungsgemeinschaft postdoctoral fellowship Mu773/1-1 to Hermann J. Müller. We thank J. Duncan, G. W. Humphreys, W. Epstein, and three anonymous reviewers for their extremely helpful comments on earlier versions of this article. Many thanks are also extended to $\mathbf{M}$. Shepherd for providing his EMDISP system and to J. M. Findlay for his help with eye movement monitoring.

Correspondence concerning this article should be addressed to $\mathrm{H}$. J. Müller, Department of Psychology, Birkbeck College, University of London, Malet Street, London, WCIE 7HX, England.
Reflexive orienting, triggered by peripheral cues, is automatic; voluntary orienting, initiated by central cues, is controlled. Jonides (1981) found that unlike controlled orienting in response to central cues, automatic orienting to peripheral cues is not affected by a secondary memory task (Experiment 1 ) or by the relative frequency with which different kinds of cue are given (Experiment 3) and cannot be voluntarily suppressed (Experiment 2). Furthermore, if the magnitude of priming is computed by summing benefits of correct cuing and costs of incorrect cuing, peripheral cues produce a greater effect than central cues.

Similar to Posner (1980), Jonides (1981) assumed that there is one mechanism responsible for movements of the mind's eye (i.e., orienting) but that this mechanism may be guided either by voluntary or by automatic control. If the distinction between automatic and controlled orienting rests only on the ways in which attentional orienting is initiated, the results of two of Jonides's (1981) experiments are unsurprising. Central symbolic cues, such as arrows, must be decoded before the spatial location that they designate can be determined. Peripheral cues convey spatial information directly because they occur at the locations at which the subsequent target signals are likely to be presented. Thus, it is not surprising that a secondary memory load (Experiment 1 ) or the relative unexpectedness (within a block of trials) of central cues, by demanding additional processing resources (see Navon, 1984), may interfere with the interpretation of symbolic central cues more than that of direct peripheral cues.

\footnotetext{
${ }^{1}$ Spatial orienting means allocation of a limited-capacity attention system to selected spatial locations. Thus, determining the locations to which spatial cues refer must occur prior to selection and orienting itself (see Duncan's, 1980b, notion of a selection schedule). Allocation of limited-capacity attention is accomplished through an orienting mechanism that is initiated by the output of the anteceding cue processing.
} 
Jonides's (1981) Experiment 2 is more interesting. It was designed to test whether observers could suppress orienting in response to peripheral and to central cues. Jonides (1981) found that when subjects were instructed to attend to the cue, reaction times (RTs) were faster to targets at cued rather than uncued locations, both with peripheral and with central cues. However, when subjects were told to ignore the cue, an advantage for cued over uncued locations occurred only with peripheral cues. Jonides (1981) concluded that it is possible to suppress orienting in response to central cues but not to peripheral cues.

However, on its own this finding does not establish that orienting in response to symbolic central cues cannot be automatic. It can be argued that attentional orienting, once triggered, proceeds automatically but that processes that antecede orienting, such as decoding of cue directionality or validity, can be voluntarily suppressed. Nevertheless, Jonides's (1981) Experiment 2 did demonstrate that peripheral cues, by contrast, cannot be prevented from activating the orienting mechanism.

In summary, the available evidence suggests that directionality of direct peripheral and symbolic central cues is determined through separate processes, but the information derived by these processes is fed into the same orienting mechanism. Processing of central cues can be suppressed, so initiation of the orienting mechanism is not obligatory.

Müller and Findlay (in press) replaced the assumption of a common orienting mechanism underlying reflexive and voluntary orienting by the proposal that there are two separate mechanisms that come into play at different times after cue onset: Peripheral cues trigger both a fast-acting reflexive (automatic) and a slower-acting voluntary (controlled) orienting mechanism. The rapid automatic mechanism has a powerful but transitory response that fades out 100-300 ms after cue onset. The controlled mechanism has a longer rise time and is less effective but shows a longer persistence. The reflexive mechanism is triggered by immediate physical properties of the cue (e.g., the abruptness of onset; see Jonides \& Yantis, 1988; Yantis \& Jonides, 1984) and is little affected by cue validity, that is, the probability with which the cue predicts the target location. The voluntary mechanism, however, requires development of a spatial expectancy (set) on the basis of the probabilistic information provided by the cue. Central cues initiate only the voluntary orienting mechanism.

Müller and Findlay's (in press) suggestion was based on differences in the time course of facilitatory (i.e., benefits for cued locations) and inhibitory (i.e., costs for uncued locations) effects produced by peripheral and central cues. ${ }^{2}$ They compared relatively large ranges of intervals between cue and target onsets (stimulus onset asynchronies; SOAs), that is, 50 to $700 \mathrm{~ms}$. With peripheral cues the peak facilitation for cued locations occurred within $150 \mathrm{~ms}$ after cue onset, was followed by a decline, between 150- and 300-ms SOAs, and sustained facilitation at the lower level; inhibition for uncued locations was strongest at short SOAs and then showed a marked reduction within $300 \mathrm{~ms}$ after cue onset. With central cues the facilitation for cued locations built up more gradually, requiring $300 \mathrm{~ms}$ to reach optimum, and was then maintained at optimum level; inhibition for uncued locations showed some initial increase and later decrease. With SOAs of less than $300 \mathrm{~ms}$, the sum of costs and benefits was greater for peripheral than for central cues. At longer SOAs peripheral and central cues had the same effects.

The finding that peripheral cues produce stronger facilitatory and inhibitory effects at short rather than at long SOAs (see also Jonides, 1981) is crucial to Müller and Findlay's (in press) argument for two separate orienting mechanisms. Note that although this is a quantitative effect, it suggests that separate mechanisms are involved. Were there only one orienting mechanism, that is, were allocation of the limitedcapacity attention system triggered on registration (direct peripheral cues) or derivation (symbolic central cues) of one common stimulus, then the action of the system ought to depend in an all-or-none fashion on the presence or absence of this stimulus. ${ }^{3}$

Müller and Findlay (in press) interpreted the stronger effect of peripheral cues at short rather than at long SOAs as evidence that reflexive orienting, occurring rapidly after cue onset, is characterized by greater resistance to interruption than voluntary orienting, which comes into play only later. Strong effects of peripheral cues were also found with singleelement displays in which targets (presented for a limited exposure duration) appeared on their own (i.e., in which nontarget locations remained empty).

When a single target occurred at an uncued location, the luminance increment associated with its onset ought to have attracted attention by the same (automatic) mechanism as the preceding brightening from the peripheral cue at the cued location. However, the strong inhibitory effect at short cuetarget SOAs suggests that when the automatic orienting mechanism is engaged by the peripheral cue, it cannot be interrupted by the onset of a target at an uncued location. The decrease in the inhibitory effect at longer SOAs can be explained if after some delay automatic orienting fades out and is replaced by the controlled mechanism. Voluntary orienting to the cued location can be interrupted by an automatic orienting response to a competing target, reducing any inhibition from an incorrect cue. If reflexive and voluntary orienting involve separate mechanisms that, nevertheless, address a common limited-capacity attention system, it is not immediately clear why voluntary orienting ought to be interrupted by a stimulus triggering a competing reflexive orienting response. However, this can easily be explained by assuming that the reflexive mechanism modulates the voluntary mech-

\footnotetext{
${ }^{2}$ Differences in time courses (i.e., rise times) between peripheral and central cuing presumably reflect differences in the time required to process the cues (see Eriksen \& Colegate, 1971; Eriksen \& Hoffman, 1972) and as such are not surprising; in contrast, differences in magnitudes of cuing effects are.

${ }^{3}$ This presupposes that the orienting mechanism is released by its proper (suprathreshold) trigger stimulus, that is, that quantitative aspects of the trigger stimulus have only little effect on the response of the mechanism. With regard to peripheral cuing, this assumption is supported by Jonides and Yantis's (1988) finding that whether or not reflexive orienting occurs depends on the presence or absence of an abrupt stimulus onset rather than on quantitative stimulus aspects such as differences in luminance or hue.
} 
anism through an inhibitory connection. To the extent that interruptability constitutes a criterion for the automaticity of a given mechanism (e.g., Jonides, 1981; LaBerge, 1981; Schneider \& Shiffrin, 1977; Shiffrin \& Schneider, 1977), reflexive and voluntary orienting can be characterized as automatic and controlled, respectively.

Whereas Müller and Findlay's (in press) two-mechanism model seems a plausible extension of Posner's (1980) and Jonides's (1981) proposals, their time course data do not however rule out alternative interpretations. One effect, the decline in facilitation at long SOAs that occurs with peripheral but not with central cues, may reflect Posner and Cohen's (1984) inhibition effect (see also Maylor, 1985; Posner, Rafal, Choate, \& Vaughan, 1985) rather than the transition from reflexive to voluntary orienting. According to Posner and Cohen (1984), "some part of the pathway from the cued location is reduced in efficiency by the [peripheral] cuing" ( $p$. 537), thus favoring the sampling of areas of the visual field at which there was no previous change in light energy. Posner and Cohen's inhibition effect has only been found with spatially uninformative cues but not when targets were more likely to occur at cued than at uncued locations. Posner and Cohen concluded that inhibition may be overlaid by facilitation produced by active orienting to the cued location. Because central cuing does not involve a change in light energy at the indicated location, no inhibitory consequences for this location are to be expected. This is consistent with Müller and Findlay's (in press) result with central cues, for which facilitation (cued locations) is maintained at optimum level at long SOAs.

A second finding that may be at variance with Müller and Findlay's (in press) two-mechanism model is that central cues, too, showed evidence of stronger inhibition at short rather than at long SOAs. This may indicate that early orienting to central cues is as resistant to interruption as is early orienting to peripheral cues.

These alternative accounts cannot be refuted from Müller and Findlay's (in press) data, because direct comparisons between their peripheral- and central-cuing conditions are limited. Either these conditions were presented one after the other, with different subjects and different threshold exposure times (Experiments 1 and 2), or crucial cue-target SOAs were not tested (Experiment 4). Experiment 1 of our study was designed to establish the time course of reflexive and voluntary orienting mechanisms by directly comparing peripheraland central-cuing conditions. This comparison was expected to show whether reflexive orienting is more effective and less interruptable than voluntary orienting and whether Posner and Cohen's (1984) inhibition effect can be suppressed by voluntary orienting. If reflexive orienting is more effective and less interruptable than controlled orienting, optimum facilitation produced by peripheral cues at short SOAs ought to be greater than facilitation that can be sustained with peripheral or central cues at long SOAs, and inhibition at short SOAs ought to be stronger with peripheral than with central cues. Furthermore, if Posner and Cohen's inhibition effect is suppressed by voluntary orienting, facilitation ought not to differ between peripheral and central cuing at long SOAs.
Experiments 2 and 3 were designed to test directly the interruptability of the two orienting mechanisms hypothesized to underly the time course data of Experiment 1. Interruptability was indexed by the degree to which orienting in response to cues was interfered with by task-irrelevant (i.e., uninformative) peripheral flashes. Unlike Jonides (1981, Experiment 2), Experiments 2 and 3 did not measure resistance to suppression but rather resistance to competition, that is, interruptability of orienting after it was initiated. If reflexive orienting is indeed automatic, it ought to be highly resistant to the competition of peripheral flashes at uncued locations. If voluntary orienting is controlled, it ought to be less resistant to competition.

\section{Experiment 1}

\section{Method}

Apparatus and materials. The stimuli were presented on a Tektronix $608 \mathrm{X}-\mathrm{Y}$ display with $\mathrm{P}-31$ phosphor. The cathode-ray tube (CRT) was controlled by a LSI-11/23 computer through a CED-502 interface; the display system used was EMDISP (Shepherd, 1984). The laboratory was dimly illuminated. Stimulus luminance was $0.343 \mathrm{~cd} /$ $\mathrm{m}^{2}$, and screen background luminance was $0.034 \mathrm{~cd} / \mathrm{m}^{2}$. Subjects viewed the CRT from a distance of $50 \mathrm{~cm}$ with their heads resting on a chin rest.

Display and timing. Figure 1 illustrates the sequence of frames presented on a given trial. Frame 1 displayed a central fixation dot and four peripheral boxes marking the possible target locations. After $500 \mathrm{~ms}$ the fixation dot was replaced by a central box containing a $\mathrm{T}$ in one of the four orthogonal orientations (Frame 2), which was presented for $1,500 \mathrm{~ms}$; this $\mathrm{T}$ was the comparison stimulus for the trial. Then the fixation dot reappeared (Frame 3), and 1,000 ms later a spatial cue indicating the likely target location was presented. The cue was either a 50-ms brightening of the outline of one of the peripheral boxes (peripheral cue; Frame 4a) or a 50-ms arrow indicator in the center (central cue; Frame 4b). In addition, baseline trials were given on which either all four boxes were brightened simultaneously (peripheral cuing) or a cross appeared in the center (central cuing); these events indicated that the target was equally likely to appear at all four locations. After variable cue-target SOAs the target stimulus, a $T$ in the same or a different (orthogonal) orientation as the comparison $\Upsilon$, was presented in one of the four boxes for a limited exposure duration; the three nontarget locations contained distractor crosses of the same size and luminance as the target. On valid trials (Frame 5a), the target appeared at the cued location. On invalid trials (Frame 5b), the target occurred at one of the uncued locations. Target eccentricity was $4.40^{\circ}$, and its size was $0.25^{\circ}$. In Frame 6, the target and distractors were terminated by contour masks (squares composed of $0.25^{\circ}$ lines).

Task. The subjects had to give three responses on a hand-held keypad: (1) a same-different response to indicate whether the orientation of the target $T$ (Frame 6) was the same as or different from that of the comparison $T$ (Frame 2); (2) a position response to indicate in which of the four boxes the target $T$ had appeared; and (3) another position response to indicate which position had been cued $(0$ on baseline trials). This third response ensured that a trial was accepted only when decoding of cue direction was successful. ${ }^{4}$ If the decoding was not successful, the trial was rejected and rerun later in the block.

\footnotetext{
${ }^{4} \mathrm{~A}$ correct cued position response to accept a trial was required because Müller (1984) observed that if central arrows are presented for brief times only, their direction may be confused (i.e., confusions between cued and diagonally opposite locations), which may lead to orienting to an uncued location and thus reduced priming effects.
} 


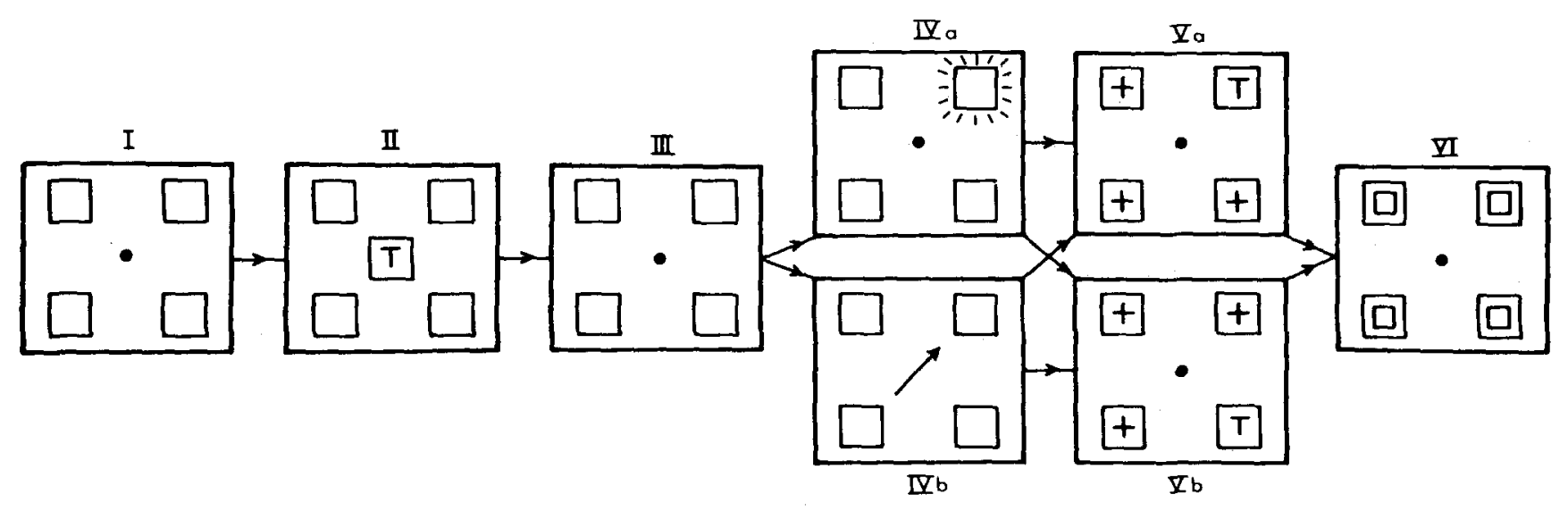

Figure 1. Sequence of frames presented on a trial.

Design and procedure. There were three variables: (a) peripheral or central cuing, (b) valid or invalid trial, and (c) cue-target SOA $(100,175,275,400,550$, or $725 \mathrm{~ms})$. The experiment was divided into two 4-hr sessions, each consisting of two blocks of peripheraland two blocks of central-cuing trials ( 360 trials per block); the order of blocks was counterbalanced across subjects and sessions. Central and peripheral cues were presented in separate blocks, rather than in random order within the same block, because expectedness of central indicators affects cue processing (Jonides, 1981). Cue validity and SOA conditions were presented in random order within a block of trials. The four orientations of target Ts were presented with equal frequency. In half the trials the targets were the same as comparison Ts; in the other half they were different. Targets appeared with equal frequency at each of the four peripheral locations. Cues were valid on half the trials and invalid on the other half. The cued position was three times as likely to contain the target as any one of the three uncued locations. The number of trials totaled 2,880 , that is, 1,440 peripheral-cuing and 1,440 central-cuing trials (6 SOAs $\times[96$ Valid Trials +96 Invalid Trials +48 Baseline Trials]).

Determination of the threshold exposure durations. At the beginning of each session, target exposure times were determined individually for each subject and separately for each cuing condition (with the order of conditions counterbalanced across sessions and subjects). The estimation procedure used was a modified Probability Estimation by Sequential Testing (PEST) adaptive staircase (Findlay, 1978), which aimed at a threshold level of $75 \%$ on baseline trials. (Threshold trials presented either a central cross or four simultaneous peripheral flashes, followed by the target at SOAs randomly chosen from the set of SOAs in Experiment 1.) This procedure was based on jointly correct same-different and position responses; that is, an error was counted if one or both responses were incorrect. The target exposure times introduced in peripheral- and in central-cuing blocks were the means of the $75 \%$ thresholds estimated for the two cuing conditions. Peripheral- and central-cuing baseline trials (with fixed exposure duration) in Experiment 1 served to provide a check on the validity and stability of the threshold estimates.

Instructions. The subjects were asked to respond as accurately as possible. They were fully briefed on all probability contingencies described earlier. They were instructed to attend to the cued location but to maintain fixation on the central dot.

Subjects. Four subjects participated in Experiment 1. Their ages ranged from 20 to 30 ; all had normal vision. Each was paid $£ 5.0$ (about \$8.75) for each 4-hr session. Three subjects had taken part in the study by Müller and Findlay (in press). One received a similar amount of practice in four preexperimental sessions. The subjects' exposure times were as follows (means of two sessions): $49.5,44.0$, 48.0 , and $46.5 \mathrm{~ms}$.

Eye-movement control. Because eye position was not monitored in Experiment 1, effects of attention on accuracy might be confounded with effects of retinal eccentricity (i.e., saccades to cued locations could be executed at longer cue-target SOAs). To rule out this possibility, Experiment 1 was repeated for all 4 subjects with the monitoring of eye movements. Design and procedure were the same as before, except that only two SOAs (175 and $725 \mathrm{~ms}$ ) were presented, and baseline trials were not included. Horizontal movement components were monitored by using a modification of Findlay's (1974) limbus-tracking technique in which a fiber-optic Y-guide is used both to illuminate the iris-sclera boundary and to detect reflected light (see Findlay, 1981, for further details). Output from the eye-movement recorder was sampled every $10 \mathrm{~ms}$, starting at cue onset and ending $150 \mathrm{~ms}$ after target onset. Samples were analyzed at the end of the trial. If a saccade was detected (velocity criterion: two successive sample differences, both in the same direction, exceeding approximately $30^{\circ} / \mathrm{s}$ ), the trial was rejected and rerun later in the block. ${ }^{s}$ This eye-movement control leaves the possibility of slow fixation drifts (e.g., Kowler \& Steinman, 1979a, 1979b). Müller and Findlay (1987), investigating eye fixation in a spatial-cuing task with the same displays as in this study, found that such drifts do indeed occur. However, only about $60 \%$ of drifts were in the cued, that is, attended, direction, and drift size was too small $\left(75 \%\right.$ were smaller than $0.375^{\circ}$, and $95 \%$, smaller than $0.625^{\circ}$ ) to have a noticeable effect on accuracy. Drifts in excess of about $1^{\circ}$ are required to affect accuracy.

Analysis. The main performance measure was the joint probability of a correct position (CP) and a correct same-different (CSD) response, $p(C P, C S D)$. Note that any bias inherent in one measure can call into question whether effects observed are attentional in nature (see Duncan, 1980a). In particular, guesses based on the knowledge that targets are more likely to occur at cued than at uncued locations (i.e., a bias in the localization task) can inflate accuracy for valid trials at the expense of invalid trials. One possible solution is to analyze the discrimination data on their own. However, detailed analyses of errors in this task (Müller \& Rabbitt, 1988) revealed that if target localization fails, the same-different response is a guess; that is, the conditional probability of a correct same-different response given an incorrect position (IP) response, $p(C S D / I P)$, is close to .5 . That is, discrimination accuracy with localization failures adds little

${ }^{5}$ Saccade probabilities averaged $.009, .013$, and .015 for Experiments 1,2 , and 3 , respectively, and did not differ between experimental conditions. 
information to that provided by discrimination accuracy with successful localization, that is, $p(C P, C S D)$. Thus, analysis of $p(C P$, $C S D)$ can be justified if $p(C S D / I P)$ is close to chance and independent of experimental variables. ${ }^{6}$

\section{Results and Discussion}

Figure 2 presents mean values of $p(C P, C S D)$ as a function of cue-target SOA, separately for central and peripheral cuing,
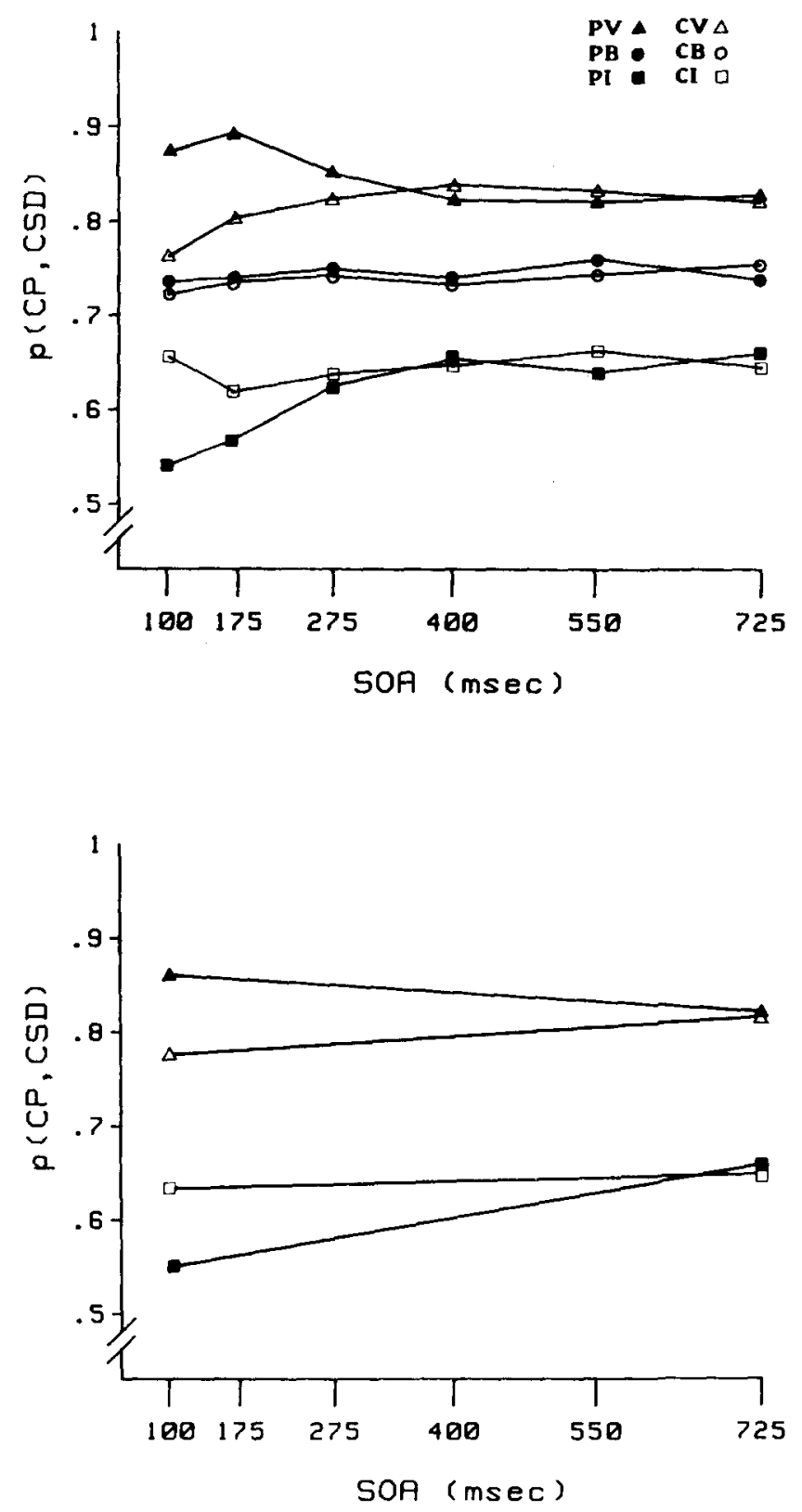

Figure 2. The probability of correct position-correct same-different responses, $p(C P, C S D)$, as a function of cue-target stimulus onset asynchrony (SOA) plotted separately for peripheral-cuing $(\mathrm{P})$ valid (V), baseline (B), and invalid (I) trials and for central-cuing (C) V, B, and I trials. (Top, data from experimental trials; bottom, data from eye-movement control trials.) valid and invalid trials, and baseline trials. Figure 2 also presents the corresponding data for the eye-movement control condition. The values of $p(C P, C S D)$ were arcsin-transformed (Winer, 1971) and evaluated in a three-way analysis of variance (ANOVA) with main terms for central or peripheral cuing, valid or invalid trial, and SOA duration. All comparisons between experimental conditions presented are based on the Tukey method.

The three-way interaction was significant, $F(5,15)=40.79$, $p<.001$, because at 100 - and 175-ms SOAs, costs plus benefits were greater for peripheral than for central cuing. SOA functions for peripheral and central cuing and for valid and invalid trials are characterized as follows:

Peripheral cuing. Valid trials (cued locations) showed a fast rise in accuracy, peaking at 175-ms SOAs; thereafter, accuracy declined toward 400-ms SOAs and then remained constant ( $p<.001$ for all comparisons between SOAs shorter and longer than $275 \mathrm{~ms}$ ). Invalid trials (uncued locations) showed an improvement between 100- and 400-ms SOAs and then remained invariant at the higher level $(p<.001$ for all comparisons between 100- to 175-ms SOAs and the longer SOAs).

Central cuing. Valid trials showed a gradual buildup in accuracy, particularly marked between 100- and 275-ms SOAs $(p<.001$ for all comparisons between SOAs shorter and longer than $175 \mathrm{~ms}$ ). Invalid trials showed some decrease between 100- and 175-ms SOAs $(p<.05$ ), followed by a tendency to increase towards 400-ms SOAs.

Valid trials. Peripheral cues showed higher accuracy at 100 - and $175-\mathrm{ms}$ SOAs than central cues at the same $(p<$ $.001)$ and at longer SOAs $(p<.01)$.

Invalid trials. Peripheral cues showed lower accuracy at 100 - and 175-ms SOAs than did central cues $(p<.005)$.

Valid and invalid trials. There are no reliable differences between peripheral and central cuing at SOAs longer than $175 \mathrm{~ms}$.

Figure 2 shows that the eye-movement control condition is characterized by the same pattern (Peripheral vs. Central Cuing $\times$ Valid vs. Invalid Trials $\times$ SOA Interaction). That is, this pattern cannot be attributed to strategic eye movements.

These results are consistent with the two-mechanism model of spatial orienting: The fast-acting mechanism triggered by peripheral cues produces a powerful facilitatory effect for cued locations (100- to $175-\mathrm{ms}$ SOAs). This mechanism is effective only for a short time, and as it fades out, facilitation for cued locations declines. However, within $275-400 \mathrm{~ms}$ after cue onset, a second mechanism comes into effect that enables attention (i.e., facilitation) for the cued position to be sustained, even though at a lower level. Central cues initiate only this second, slower-acting mechanism.

\footnotetext{
${ }^{6}$ Analyses of variance of arcsin probability of correct same-different response given incorrect position response, $p(C S D / I P)$, for Experiments 1,2 , and 3 did not reveal any significant effects. The overall values of $p(C S D / I P)-.533, .535$, and .528 , respectively-differed only little from chance, thus justifying the use of probability of correct position-correct same-different responses, $p(C P, C S D)$, as a performance measure.
} 
Maximum facilitation achieved by peripheral cues (100- to 175-ms SOAs) is greater than that achieved by central cues (at 400- to 725-ms SOAs). This is consistent with the idea that the fast-acting, reflexive mechanism triggered by peripheral cues is absolutely more effective than the slower-acting, voluntary mechanism initiated by central cues. At longer SOAs peripheral and central cues produce equal facilitation. This suggests that whether leading cues are peripheral or central, they involve the same, voluntary mechanism to sustain attention for periods of time longer than $400 \mathrm{~ms}$. The finding that facilitation from peripheral cuing is as great as that from central cuing at longer SOAs also indicates that this second mechanism overlays any inhibitory consequences of peripheral cues on cued locations (i.e., Posner \& Cohen's, 1984 , inhibition effect is absent).

The two-mechanism model receives further support from the pattern of inhibition for uncued locations. With peripheral cues maximum facilitation for cued locations is associated with strong inhibition for uncued locations (100- to $175-\mathrm{ms}$ SOAs), which can be compensated for only as the reflexive mechanism is replaced by the voluntary mechanism. With central cues there is an initial increase in inhibition for uncued locations (see also Jonides, 1981, Experiment 3), followed by a nonsignificant tendency for inhibition to decrease (significant in Experiment 2 of Müller \& Findlay, in press). This suggests that developing a spatial expectancy (at short SOAs) is a more resource-demanding process than maintaining it (at longer SOAs; see also McLean \& Shulman, 1978). But initial inhibition produced by peripheral cues is much stronger than that obtained with central cues, which indicates that the reflexive mechanism triggered by peripheral cues is more resistant to interruption by a target at an uncued location calling for a competing orienting response. Thus, the claim for a fast-acting, reflexive orienting mechanism that is more resistant to interruption (by stimuli at unattended locations) than the slower-acting, voluntary mechanism explains both the greater facilitation (on valid trials) and the greater inhibition (on invalid trials) from peripheral cuing at short SOAs.

\section{Experiment 2}

If attention for the cued location is maintained at longer SOAs by the voluntary mechanism, orienting ought to be interruptable by a competing stimulus, in particular if that stimulus triggers a reflexive orienting response. Experiment 2 was designed to measure the degree to which sustained, voluntary orienting, initiated through central cues, can be interrupted by task-irrelevant peripheral flashes.

On every trial (except for the baseline), a 300-ms central arrow that indicated the likely target location (Frame 4 of the display cycle) was presented, and this cue was followed by the target after varying SOAs $(600,900$, or $1200 \mathrm{~ms})$. In one third of the trials, the central cue only was presented. In the other two thirds there was an additional 50-ms brightening of the outline of one of the peripheral boxes that occurred at a fixed interval of $500 \mathrm{~ms}$ after the central cue onset (i.e., the SOAs between flash and target were 100,400 , and $700 \mathrm{~ms}$ ). Cuetarget SOAs of $600 \mathrm{~ms}$ and longer were introduced to allow voluntary orienting to the cued location to be reached at flash onset. The flash was spatially uninformative; that is, it did not alter the probability with which the target occurred at the location indicated by the arrow cue. Thus, in order to maximize overall performance, the subjects had to maintain orientation to the cued location, regardless of the location of the flash; there was competition between cue and flash whenever the flash occurred at an uncued location.

In summary, Experiment 2 involved three conditions, which are illustrated in Figure 3: (1) The central cue only (C)

\section{Central cue only}

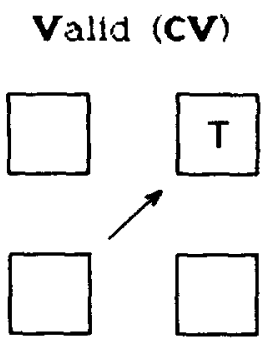

Invalid $(\mathrm{CI})$

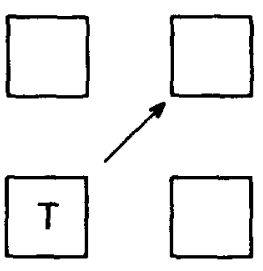

Target At Flashed Location

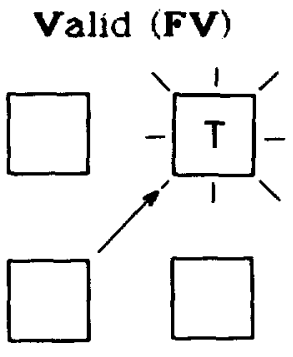

Invalid (FI)

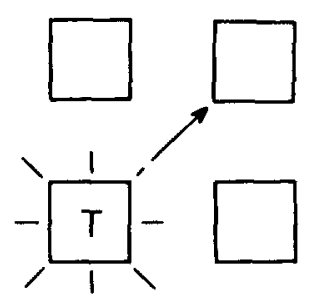

Target At Other Than Flashed Location

$$
\text { Valid (ov) }
$$

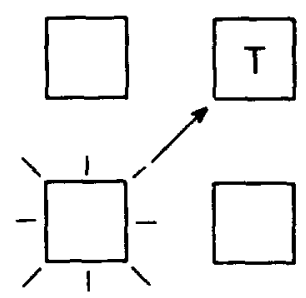

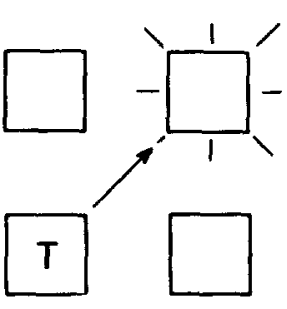

Invalld (OIb)

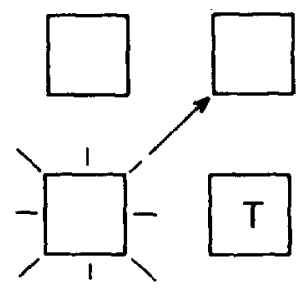

Figure 3. Central cue only, target at flashed location, and target at other than flashed location conditions. 
condition provided a baseline measure for valid (CV) and invalid (CI) trials; (2) in the target at flash location (F) condition, the target occurred at the location of the flash, which might previously have been cued or not cued (FV and FI); (3) in the target at other than flash location (O) condition, the target appeared at an unflashed location, which might have been cued or not cued (OV and $\mathrm{OI}$ ). Note that OI trials can be broken down further according to the relation between cued and flashed locations: The target could occur at one of the other-than-flashed, uncued locations with cued and flashed locations being either identical (Ola) or not (OIb).

The expectations for the critical condition in which cue and flash compete (i.e., with a flash at an uncued location) were as follows: There would be a loss in accuracy for OV trials at short SOAs, because voluntary orienting to the cued location is interrupted by a fast-acting, reflexive orienting response to the flash. At longer SOAs accuracy for OV trials would rise again, as attention is voluntarily reoriented to the cued location. For the FI trials it was expected that there would be an accuracy gain at short SOAs, because of the reflexive orienting response to the flash. At longer SOAs this advantage would be lost again because of the reorientation of attention to the cued location. That is, the expectation was for a trade-off between cued (OV) and flashed locations (FI), where gains and losses might occur relative to the corresponding $C$ conditions.

\section{Method}

Unless otherwise mentioned, the method was the same as in Experiment 1.

Subjects and apparatus. The displays were presented on a Hewlett Packard $1321 \mathrm{X}-\mathrm{Y}$ monitor (P-31 phosphor), which was controlled by an Alpha (LSI/2OG) computer. Subjects viewed the CRT from a distance of $115 \mathrm{~cm}$, which resulted in the same angular sizes and eccentricities of stimuli as in Experiment 1. Four subjects, all highly practiced with this task (Müller $\&$ Rabbitt, in press), participated in Experiment 2: Their ages ranged from 20 to $28 ; 2$ had normal vision, and 2 had corrected-to-normal vision. Each was paid $f 4.0$ (about $\$ 7.00)$ per session.

Design and procedure. There were three main variables: (a) $\mathrm{C}, \mathrm{F}$, or O condition, (b) valid or invalid trials, and (c) SOA duration (600, 900 , and 1,200 ms between cue and target, equivalent to 100,400 , and $700 \mathrm{~ms}$ between flash and target). Baseline trials, in which a spatially uninformative cross was presented, were combined with the $\mathrm{C}, \mathrm{F}$, and $\mathrm{O}$ conditions in the same way as valid and invalid trials.

Table 1 summarizes the design of Experiment 2. The ratio between trials in the $F$ and $O$ conditions was $1: 3$; the probability with which the target occurred at flashed locations was $1: 4$, so flashed locations and target locations were probabilistically unrelated. Flashes did not

Table 1

Design of Experiment 2: Number of Trials per Cue-Target Stimulus Onset Asynchrony

\begin{tabular}{lccc}
\hline \multicolumn{1}{c}{ Condition } & Valid & Baseline & Invalid \\
\hline Central cue only & 96 & 48 & 96 \\
$\begin{array}{l}\text { Target at flashed location } \\
\text { Target at other than flashed }\end{array}$ & 48 & 24 & 48 \\
$\quad$ location & 144 & 72 & $4^{\mathrm{a}}, 96^{\mathrm{b}}$ \\
\hline
\end{tabular}

a Flash at cued location. ${ }^{\mathrm{b}}$ Flash at uncued location. change the information about likely target locations provided by the arrow cues. Furthermore, each of the four locations was equally likely. to contain the target, equally likely to be cued, and equally likely to be flashed. The total number of trials was 2,160 , which were presented in two 4-hr sessions of 1,080 trials. All conditions were presented in randomized order.

At the beginning of each session, 75\%-threshold exposure durations for baseline trials (without flash) were determined for each subject. The 4 subjects' exposure times (means of two sessions) were: 34.0, $30.0,36.5$, and $35.5 \mathrm{~ms}$, respectively. Subjects were told that on cue plus flash trials, the flash would not alter the spatial information provided by the central arrow. In order to maximize overall performance, they ought therefore to ignore the flash and to attend to the cued location.

Eye-movement control. To rule out the possible confounding of attentional and retinal eccentricity effects, Experiment 2 was repeated with the 4 subjects of Experiment 1, and eye movements were monitored. The method was the same as before, except that only two SOAs (600 and $1,200 \mathrm{~ms}$ ) were presented and central cue only and baseline trials were not included (see Footnote 5).

\section{Results and Discussion}

The results are presented in three subsections: (a) Cueincompatible flashes (a trade-off in attention between $\mathrm{OV}$ and FI trials; (b) effects of flashes in $F$ and $O$ conditions in comparison with the $\mathrm{C}$ conditions; and (c) effects of cuecompatible and -incompatible flashes in Ola and OIb trials. The values of arcsin $p(C P, C S D)$ were subjected to two ANOVAS, the first with main terms for $\mathrm{C}, \mathrm{F}$, or $\mathrm{O}$ condition, valid or invalid trials, and $\mathrm{SOA}$, and the second with main terms for $\mathrm{CI}, \mathrm{Fl}$, OIa, or OIb condition and SOA. The first ANOVA of valid and invalid trials treated OI trials as a unitary condition. Only the second ANOVA of invalid trials treated OI trials as two separate conditions, according to whether the flash occurred in an OIa or in an OIb trial. The effects revealed by these ANOVAs were used for comparisons (Tukey method) between individual conditions of interest to the experimental questions.?

Cue-incompatible flashes. Figure 4 presents mean values of $p(C P, C S D)$ as a function of cue-target SOA for the critical conditions in which there was competition between flash and cue, that is, in OV, FI, and OIb trials. Figure 4 also presents the same comparison for the eye-movement control group.

The most important finding of Experiment 2 is that when cue and flash competed (i.e., when flashes occurred at an uncued location), accuracy in OV trials was markedly reduced, $100 \mathrm{~ms}$ after flash onset (600-ms cue-target SOAs),

\footnotetext{
${ }^{7}$ The first analysis of variance revealed the following significant effects (among other less important effects): main effect of central cue only $(\mathrm{C})$, target at flashed location $(\mathrm{F})$, and target at other than flashed location $(O)$ conditions, $F(2,6)=83.04, p<.001$; main effect for valid and invalid trials, $F(1,3)=276.11, p<.001 ; C, F$, and $O$ Conditions $\times$ Valid and Invalid Trials $\times$ Stimulus Onset Asynchrony (SOA) interaction, $F(4,12)=4.66, p<.025$. The second analysis revealed a significant main effect of $\mathrm{CI}, \mathrm{FI}$, OIa, and OIb trials (I indicates an invalid trial of the condition; a, flash at cued location; and $\mathrm{b}$, flash at uncued location), $F(3,9)=45.17, p<.001$, and a significant $\mathrm{CI}$, FI, OIa, and OIb Trials $\times$ SOA interaction, $F(6,18)=$ $77.04, p<.01$.
} 
whereas accuracy in FI trials was enhanced. That is, at 600ms SOAs, the standard advantage of spatial cuing is abolished: Accuracy was greater $(p<.001)$ in FI trials than in OV trials. Concomitantly, accuracy in FI trials was greater $(p<.001)$ than that in OIb trials, even though uncued locations were equally likely to contain a target. Note that accuracy in OV trials was greater than that in OIb trials, $100 \mathrm{~ms}$ after flash onset $(600$-ms SOAs; $p<.001)$; that is, among other-thanflashed locations, flashes were less detrimental to accuracy for likely (OV trials) than for unlikely (OIb trials) locations.

This pattern is consistent with the idea that voluntary (sustained) orienting in response to the central cue is interrupted by a reflexive orienting response triggered by a competing peripheral flash. The flash rapidly captures attention, inhibiting accuracy for other than flashed, cued and uncued locations. This effect indicates that an abrupt discontinuity in light energy is a proper trigger stimulus for a reflexive orienting response that may not be prevented, though possibly modified, by voluntary control (see later discussion).

As SOA duration became longer, accuracy declined in $\mathrm{FI}$ trials and increased in OV trials. Within 100-400 ms after flash onset, functions for flashed and cued locations intersect, reestablishing the standard advantage of spatial cuing. Functions for uncued locations converge, as accuracy declined in FI trials and increased, within $100-400 \mathrm{~ms}$ after flash onset, in Olb trials.

The trade-off in attention between flashed and cued locations is consistent with the idea that the period for which the reflexive orienting mechanism is active is transitory. Within $100-400 \mathrm{~ms}$ after its onset, reflexive orienting to the flash became increasingly interruptable by voluntary control, that is, voluntary reorientation of attention to the location indicated by the central cue.

As can be seen in Figure 4, the eye-movement control group showed the same pattern. That is, the trade-off in attention between FI trials and OV trials was not produced by strategic eye movements (e.g., by first saccading to the flashed location and then back to the cued location).

Effects of flashes in comparison with the central cue only condition. Figure 5 shows how the effects of the flash compare with the $C$ conditions. This figure presents mean values of $p(C P, C S D)$ as a function of cue-target SOA, separately for $C, F$, and $O$ conditions and for valid, baseline, and invalid trials. Note that in Figure 4, OI trials include all trials in which targets appeared at other than flashed, uncued locations, no matter whether flashes occurred at cued (Ola) or uncued (OIb) locations (for differences between these conditions, see Figure 6).

As can be seen from the $\mathrm{C}$ condition (left side of Figure 5), accuracy was higher in CV trials than in CI trials at all SOAs $(p<.001)$, a pattern in agreement with the central-cuing condition of Experiment 1. Thus, when subjects did not know in advance whether or not a flash would occur on a given trial, spatially informative arrow cues produced the standard cuing effects.

With reference to the $\mathrm{C}$ condition, the effect of the flash appeared rather stereotyped: In the $F$ condition (middle of Figure 5), accuracy was enhanced $100 \mathrm{~ms}$ after flash onset (i.e., at 600-ms SOAs), and in the $\mathrm{O}$ condition (right side), accuracy was impaired-no matter whether flashes occurred at cued or at uncued locations. Note, however, that within the $\mathrm{F}$ and $\mathrm{O}$ conditions, costs (uncued locations) plus benefits
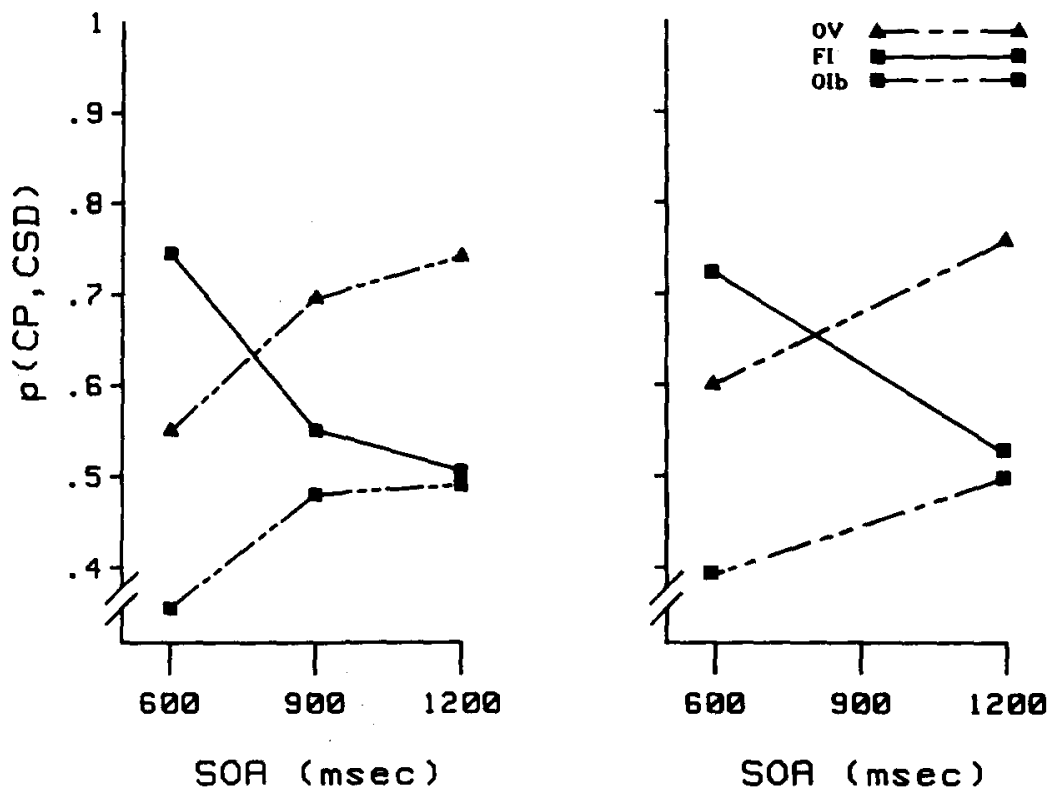

Figure 4. Cue-incompatible flashes: The probability of correct position-correct same-different responses, $p(C P, C S D)$, as a function of cue-target stimulus onset asynchrony (SOA) plotted separately for target at other than flashed location, valid (OV), target at flashed location, invalid (FI), and target at other than flashed location, invalid, flash at uncued location (Olb) trials. (Left, data from experimental trials; right, data from eye-movement control trials.) 


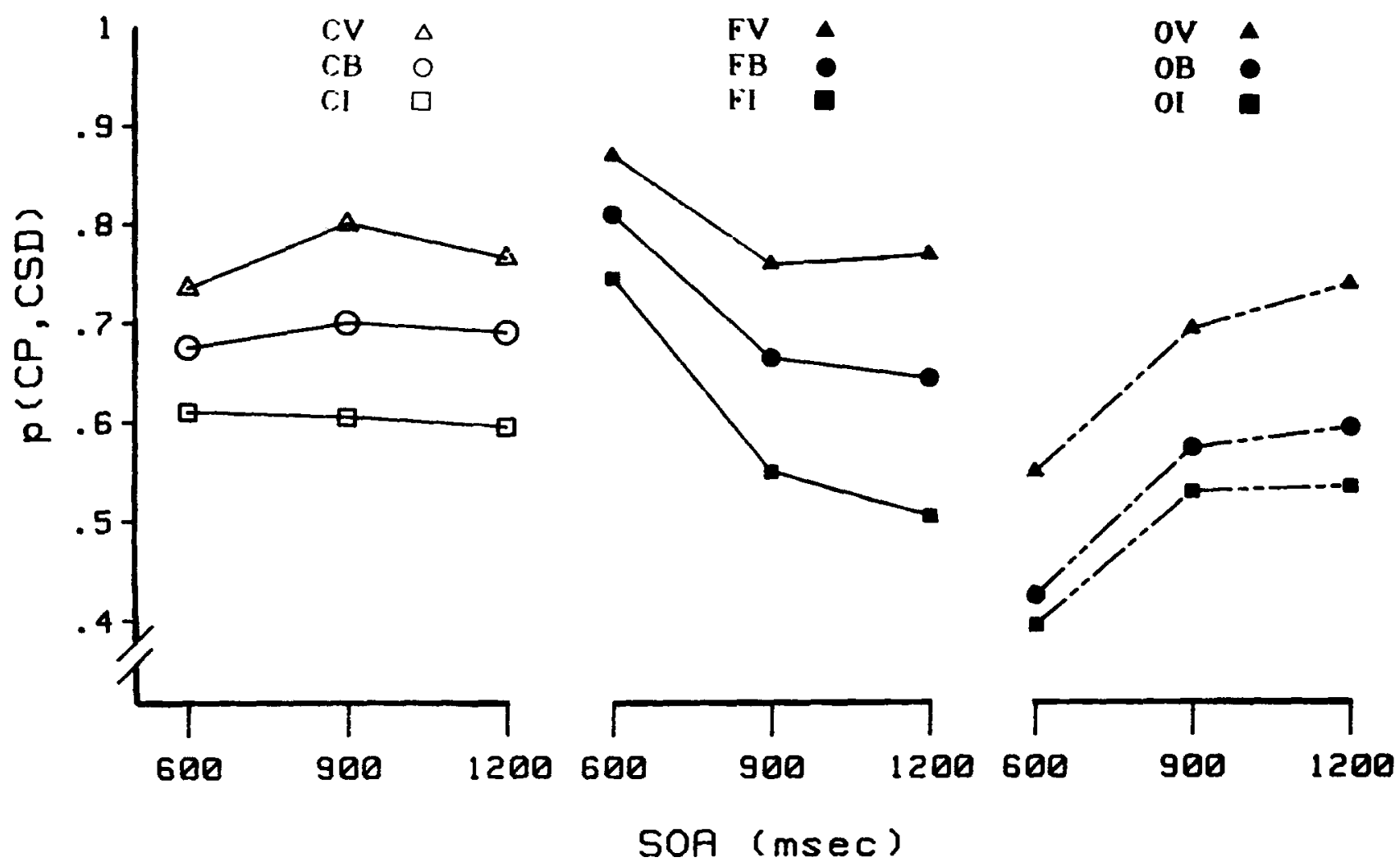

Figure 5. The probability of correct position-correct same-different responses, $p(C P, C S D)$, as a function of cue-target stimulus onset asynchrony (SOA) plotted separately for central cue only (C) valid (V), baseline (B), and invalid (I) trials (left), for target at flashed location (F) V, B, and I trials (middle), and for target at other than flashed location $(\mathrm{O}) \mathrm{V}, \mathrm{B}$, and I trials (right).

(cued locations) were significant $(p<.001)$, even at 600 -ms SOAs. Thus, the flash, $100 \mathrm{~ms}$ after its onset, did not affect the standard advantage for cued over uncued locations.

Three observations are of particular interest in this context. When the flash occurred at an uncued location, accuracy in OV trials was less inhibited, $100 \mathrm{~ms}$ after flash onset, than was accuracy in OI trials (OV-OI comparison, $p<.001$ ). When the flash occurred at the cued location, accuracy in FV trials was more facilitated, shortly after the flash, than in the $\mathrm{CV}$ condition (FV-CV comparison, $p<.001$ ). Facilitation for flashed locations was more marked when a flash occurred at a likely (FV trials) than at an unlikely (FI trials) location (FV-FI comparison, $p<.001$ ).

Thus, it appears that facilitatory effects produced by uninformative flashes can add to the facilitation produced by voluntary orienting to the cue, whereas voluntary orienting to the cue can subtract from inhibitory effects of flashes (see General Discussion for an elaboration of this point).

Effects of cue-compatible and-incompatible flashes in OIa and $\mathrm{OIb}$ trials. In Figure 5, OI trials were presented as a unitary condition. However, flashes might have different effects in these trials depending on whether flashes occurred at cued (Ola) or at uncued (OIb) locations. Figure 6 presents $p(C P, C S D)$ separately for OIa and Olb conditions and also, to make comparisons easier, for FI and CI trials.
Accuracy in OIa and OIb trials was markedly reduced 100 ms after flash onset (600-ms SOAs): The reflexive orienting response triggered by the flash was associated with a rapid buildup of inhibition at other than flashed locations. This inhibitory effect shows a remarkable difference according to whether the flash occurred at a cued (OIa trials) or at an uncued (Olb trials) location: In an OIa trial, inhibition was less marked $(p<.001)$ than in an OIb trial. Both conditions improved within $100-400 \mathrm{~ms}$ after flash onset. However, accuracy in OI trials reached a comparable level to that in $\mathrm{CI}$ trials only for the Ola condition (OIa-CI comparisons at 600ms SOA, $p<.001$; at longer SOAs, $n s)$. In the OIb condition, accuracy stayed well below this level (i.e., the advantage of Ola over Olb persists at longer SOAs; $p<.001$ at $900-$ and 1,200-ms SOAs).

This pattern suggests that two elements contributed to the inhibitory effect for uncued locations: The first was fastacting, passive inhibition caused by the capture of attention by the flash, no matter whether it occurred at the cued (OIa) or at an uncued location (OIb). This inhibition could be compensated for as the power of the flash to engage the reflexive orienting mechanism faded out within $100-400 \mathrm{~ms}$ after flash onset. The second was active inhibition that occurred only with a flash at an uncued location (OIb). This is caused by the additional resource demands of the voluntary 


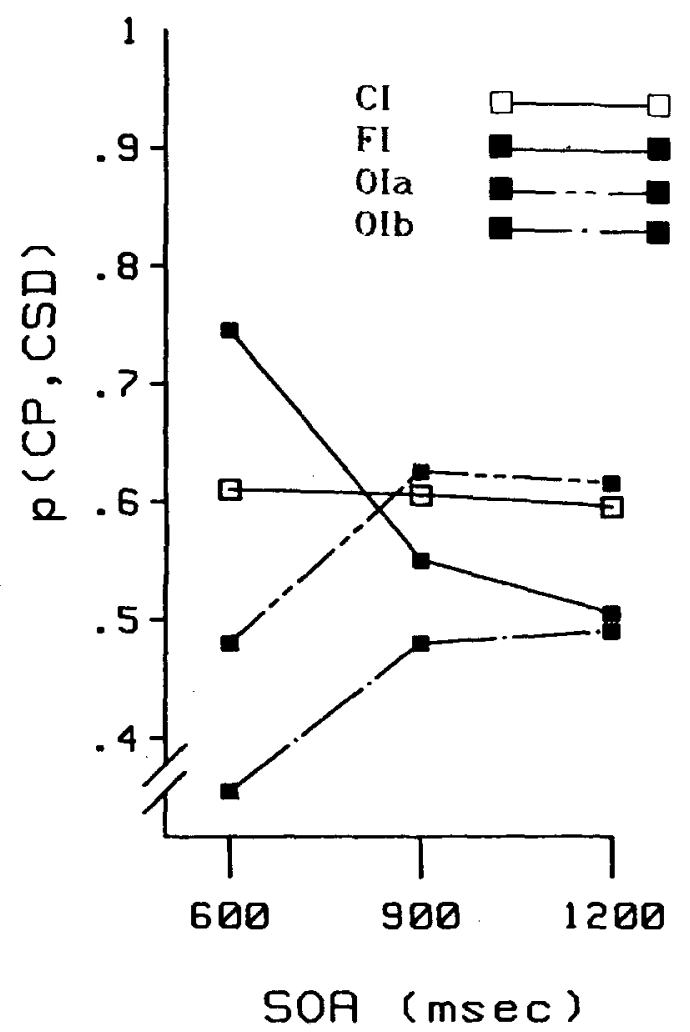

Figure 6. The probability of correct position-correct same-different responses, $p(C P, C S D)$, as a function of cue-target stimulus onset asynchrony (SOA) plotted separately for target at other than flashed, invalid (OI) trials with cue-compatible (a) or cue-incompatible (b) flashes, for central cue only, invalid (CI) trials, and for target at flashed location, invalid (FI) trials.

orienting mechanism, which guides reorientation of attention to the cued position. Because active inhibition is time-locked to the voluntary reorientation of attention, it can outlast passive inhibition, which is time-locked to the transitory reflexive orienting response. The additional resource demands with a flash at an uncued location may arise because the spatial expectancy for the likely location needs to be renewed or consolidated. With a flash at the cued position, no such demands arise because the flash may itself renew the spatial expectancy, and there is no need to reorient attention.

\section{Experiment 3}

Experiment 3 was designed to measure the degree to which the fast-acting reflexive and the delayed-acting voluntary mechanism underlying orienting to peripheral cues can be interrupted by task-irrelevant (uninformative) peripheral flashes. If the reflexive orienting mechanism is indeed characterized by greater automaticity, it ought to be less interruptable by a competing flash than the voluntary orienting mechanism.

A trial began with the presentation of a peripheral cue, that is, a 50-ms brightening of the outline of one of the four boxes indicating the likely target location, which was then followed after varying SOAs by a target. In one condition only the peripheral cue was presented, and in the other conditions an additional flash occurred at varying intervals after the onset of the peripheral cue (cue-flash onset asynchronies; CFOAs). This flash was spatially uninformative.

Thus, Experiment 3 involved three conditions: (a) The peripheral cue only (P) condition, (b) the target at flashed location (F) condition, and (c) the target at other than flashed location $(\mathrm{O})$ condition. The SOAs between cue and target $(\mathrm{P}$ condition) and between flash and target ( $\mathrm{F}$ and $\mathrm{O}$ conditions) were 100,300 , and $500 \mathrm{~ms}$ so that the time course of orienting in response to peripheral cues and flashes could be mapped out. CFOAs were 100,300 , and $500 \mathrm{~ms}$; that is, the flash occurred during fast-acting (100- or 300-ms CFOAs), reflexive orienting or during slow-acting (300- or 500-ms CFOAs), voluntary orienting to the cued location. SOAS of $100-\mathrm{ms}$ were just long enough to avoid apparent movement from the cued to the flashed location when peripheral cue and flash occurred at different locations and to allow cued and flashed locations to be clearly distinguished.

With cue-incompatible flashes occurring at uncued locations, it was expected that there would be losses in accuracy in OV trials at 100-ms flash-target SOAs, with the magnitude of losses depending on CFOA: At 100-ms CFOAs, losses would be less marked than at longer CFOAs because the reflexive orienting mechanism is more resistant to competition than the voluntary mechanism. At longer SOAs priority for the cued location would be reestablished (voluntary reorientation of attention).

Likewise, it was expected that there would be accuracy gains in FI trials at 100 -ms flash-target SOAs, with the magnitude of gains depending on CFOA. At 100-ms CFOAs the gains would be less marked than at longer CFOAs because the reflexive orienting mechanism is initially engaged by the peripheral cue. At longer SOAs advantages for flashed locations would be lost again because attention is strategically reoriented to cued locations.

\section{Method}

The general method of Experiment 3 was the same as in Experiment 2 . The variations were as follows.

Design and procedure. Experiment 3 involved four main variables: (a) CFOA (100, 300, and $500 \mathrm{~ms}$ ), (b) P, F, and O conditions, (c) valid and invalid trials, and (d) SOA duration $(100,300$, and 500 $\mathrm{ms})$. Note that the term $S O A$ refers to the delay between the onset of the peripheral cue ( $P$ condition) or of the peripheral flash ( $F$ and $O$ conditions) and target onset. Baseline trials, which presented a spatially uninformative, simultaneous brightening of all four boxes, were combined with the $F$ and $O$ conditions in the same way as valid and invalid trials. Furthermore, in the $P$ baseline condition (warning signal only), the target occurred not only at SOAs of 100,300 , or $500 \mathrm{~ms}$ but also at SOAs of 700 or $900 \mathrm{~ms}$ to provide a baseline measure without flash for the $F$ and $O$ conditions. The design of Experiment 3 is summarized in Table 2. The total number of trials was 2,976, which were presented in four 2 -hr sessions of 744 trials.

Subjects. Seven subjects participated in Experiment 3; their ages ranged from 19 to $29 ; 4$ had normal vision, and 3 had corrected-to-

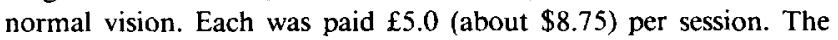
subjects having no prior experience with the task participated in 4-6 
Table 2

Design of Experiment 3: Number of Trials per Cue-Target Stimulus Onset Asynchrony (Peripheral Cue Only Condition) or per Cue-Flash Onset Asynchrony and Flash-Target Stimulus Onset Asynchrony (Target at Flashed Location and Target at Other than Flashed Location Conditions)

\begin{tabular}{lccc}
\hline \multicolumn{1}{c}{ Condition } & Valid & Baseline & Invalid \\
\hline Peripheral cue only & 96 & 48 & 96 \\
Target at flashed location & 24 & 12 & 24 \\
$\begin{array}{l}\text { Target at other than flashed } \\
\text { location }\end{array}$ & 72 & 36 & $24^{\text {a }}, 48^{\text {b }}$ \\
\hline
\end{tabular}

${ }^{a}$ Flash at cued location. ${ }^{b}$ Flash at uncued location.

practice sessions. The following times were introduced for the subjects (means of four sessions, based on threshold estimates for 5 baseline trials): $45.0,51.5,39.0,47.5,53.5,50.0$, and $50.5 \mathrm{~ms}$.

Eye-movement control. To rule out possible effects of eye movements on accuracy, Experiment 3 was repeated with the 4 subjects of Experiment 1, and eye movements were monitored. The methodology was the same as before, except that only two CFOAs (100 and $500 \mathrm{~ms}$ ) were presented and $P$ and baseline trials were not included (see Footnote 5).

\section{Results and Discussion}

The results are organized in three sections: (a) Cue-incompatible flashes (trade-offs in attention between $\mathrm{OV}$ and FI trials), (b) cue-compatible and -incompatible flashes (effects in OIa and OIb trials), and (c) cue-compatible flashes (effects in $\mathrm{FV}$ and Ola trials). The values of $\arcsin p(C P, C S D)$ were evaluated in two ANOVAs, the first with main terms for CFOA, $F$ or $O$ condition, valid or invalid trial, and SOA, and the second with main terms for CFOA, FI, OIa, or OIb trial, and SOA. These ANOVAS were used for individual comparisons (Tukey method) between conditions of theoretical interest. ${ }^{8}$

Cue-incompatible flashes. Figure 7 presents mean values of $p(C P, C S D)$ as a function of CFOA and flash-target SOA for the critical conditions in which there was competition between cue and flash, that is, in OV, FI, and OIb trials. Figure 7 also presents the same comparison for the eyemovement control group.

There were substantial losses in accuracy in OV trials and marked gains in FI trials $100 \mathrm{~ms}$ after the onset of a flash at an uncued location (100-ms SOAs); there were also losses in OI trials. That is, a flash at an uncued location rapidly captured attention, enhancing accuracy for the flashed location and reducing it for the cued location and also for other than flashed, uncued locations.

All CFOAs showed this pattern at 100-ms SOAs, but the magnitudes of gains for flashed and losses for cued locations depended on CFOA: They were less marked for 100-ms than for 300- and 500-ms CFOAs. At 100-ms CFOAs accuracy did not differ between FI and OV t.ials, but at $300-$ and $500-\mathrm{ms}$ CFOAs, it was higher $(p<.001)$ for FI than for OV. That is, 100-ms after flash onset (100-ms SOAs), accuracy in FI trials is slightly higher at 300 - and 500 -ms CFOAs than at $100-\mathrm{ms}$ CFOAs ( $n s)$; and accuracy in OV trials is lower at 300- and 500 -ms than at 100 -ms CFOAs $(p<.25)$. Similarly, accuracy in OIb trials is slightly lower at longer than at 100-ms CFOAs (ns).

This pattern is consistent with the idea that when a flash occurred at an uncued location during reflexive orienting to the peripheral cue (100-ms CFOA), its ability to capture attention and to inhibit other than flashed locations was reduced, in comparison with flashes that occurred during voluntary orienting in response to the spatial information of the cue (300- to 500-ms CFOAs). Thus, the reflexive orienting mechanism shows greater resistance against the competition of a peripheral flash (itself calling for a reflexive orienting response) than the voluntary mechanism. Note, however, that the difference is only a gradual one; that is, there is a substantial trade-off with 100-ms CFOAs. The trade-offs for longer CFOAs are as large as that in Experiment 2 (see Figure 4), which indicates that at longer cue-target SOAs, orienting is maintained by the same voluntary mechanism, whether cuing is peripheral or central.

With increasing time between flash and target (see 300- and 500-ms SOAs in Figure 7), functions for FI and OV trials intersect, as accuracy improved in OV trials and declined in FI trials. Also, functions for FI and OIb trials converge because of losses in accuracy in FI trials and gains in OIb trials (between 100- and 300-ms SOAs).

This pattern agrees with the idea that as the power of the flash to engage the reflexive orienting mechanism fades out (with increasing flash-target SOA), priority for the cued position is reestablished through voluntary orienting. This reorientation of attention to the cued location (OV trials) is associated with a substantial decline in accuracy for the flashed location (FI trials). With the fading of reflexive orienting to the flash, some enhancement (i.e., recovery from initial, passive inhibition) can also occur for other-thanflashed uncued locations (OI trials; i.e., targets at these positions become more likely to call for an orienting response).

The eye-movement control group shows the same differential effect between 100-ms and 500-ms CFOAs as the other group (see Figure 7). Thus, the trade-off in attention between FI and OV trials and the modifying effect of CFOA on this trade-off cannot be attributed to strategic saccadic eye movements, in particular, to saccades from the cued to the uncued, flashed and back to the cued location being more likely with longer than with short CFOAs.

Effects of cue-compatible and-incompatible flashes in OIa and $O I b$ trials. As in Experiment 2, flashes might have a

\footnotetext{
${ }^{8}$ The first analysis of variance revealed the following effects (among other less important effects): main effect of cue-flash onset asynchrony (CFOA), $F(2,12)=26.51, p<.001$; main effect of target at flashed location (F) and target at other than flashed location $(O)$ conditions, $F(1,6)=114.53, p<.001$; main effect of valid and invalid trials, $F(1,6)=81.13, p<.001$; CFOA $\times$ Valid and Invalid Trials interaction, $F(2,12)=18.61, p<.001 ; \mathrm{CFOA} \times$ Valid and Invalid Trials $\times$ Stimulus Onset Asynchrony (SOA) interaction, $F(4$, $24)=2.65, .075>p>.050$. The second analysis revealed (among others) the following effects: main effect of FI, OIa, and Olb (I indicates invalid trials of the condition; a, flash at cued location; and $\mathrm{b}$, flash at uncued location), $F(2,12)=30.27, p<.001$; CFOA $\times \mathrm{FI}$, Ola, and OIb Trials $\times$ SOA interaction, $F(8,48)=2.12, .075>p>$ .050 .
} 


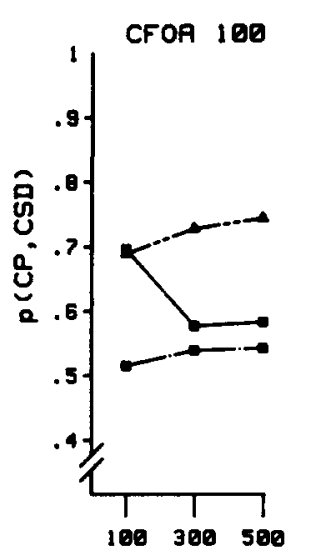

CFOA 360
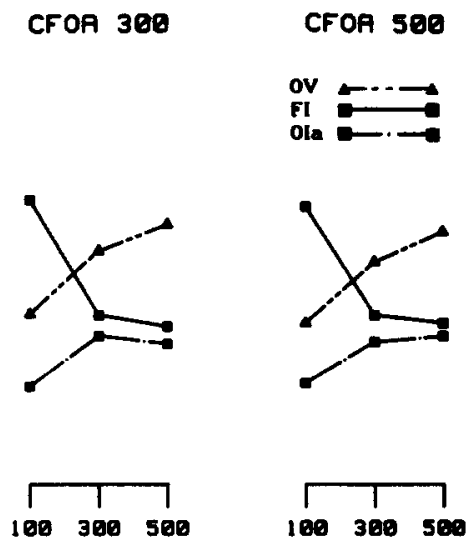

SOA (msec)

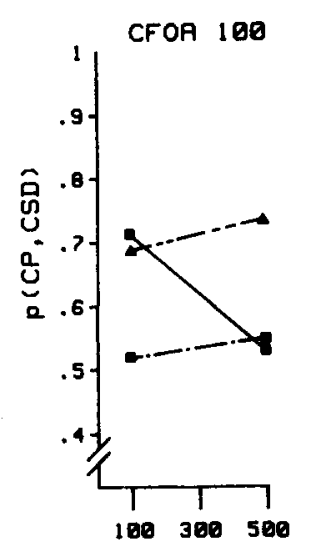

CFOA 500
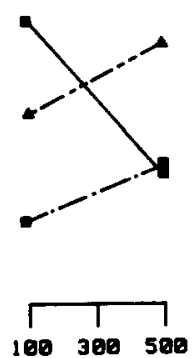

SOR (msec)

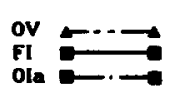
SOAs, accuracy is slightly higher in FI trials and somewhat lower in OIb trials with 300 - and 500-ms CFOAs than with 100-ms CFOAs. In other words, flashes at uncued locations are more effective at long than at short CFOAs. For a flash at cued location, at 100-ms SOAs, accuracy is slightly lower in OIa trials with a 100 -ms CFOA than with 300 - and 500-ms CFOAs. That is, unexpectedly, flashes at cued locations appear less effective at long CFOAs.

Effects of cue-compatible flashes in FV and OIa trials. The reduced effectiveness of flashes occurring at cued locations at longer CFOAs is presented in more detail in Figure 9. This figure illustrates the comparison between $\mathrm{FV}$, Ola, and $\mathrm{P}$ baseline trials (plotted as a function of cue-target SOA). Note that in Figure 9, $\mathrm{P}$ condition, valid and invalid trials are presented at the left as CFOA 0 conditions; that is, these conditions can be regarded as cue plus flash conditions with the flash occurring simultaneously with the cue (i.e., CFOA of $0 \mathrm{~ms}$ ) at the cued location.

The $\mathrm{P}$ condition showed the typical pattern associated with peripheral cuing. In FV trials, there is powerful facilitatory effect at 100-ms SOAs, followed by a decline and sustained facilitation at 300 - and 500 -ms SOAs. In Ola trials, there is an initial inhibitory effect, followed by some enhancement within $300 \mathrm{~ms}$ after cue onset. With a flash at the cued location, the pattern is essentially the same for $100-\mathrm{ms}$ CFOAs.

However, there is a change in this pattern with 300 - and 500-ms CFOAs: Accuracy for uncued locations remains invariant across SOA at a level comparable to that at 300 - and 500-ms SOAs with 0- and 100-ms CFOAs (i.e., accuracy is not reduced at 100 -ms in comparison with longer SOAs). In contrast, accuracy for cued locations is generally reduced in comparison with that with 0 - and $100-\mathrm{ms}$ CFOAs. Note the intersection between FV and $P$ baseline trials with 300 - and 500-ms CFOAs.

That is, at 300- and 500-ms CFOAs, flashes at cued locations are associated with (a) an SOA-independent inhibitory effect for stimuli at cued locations and (b) reduced inhibition at $100-\mathrm{ms}$ SOAs for stimuli in Ola trials. Thus, costs plus benefits decrease between 100 - and 300 -ms SOAs, with all CFOAs, and also between short (0- to $100-\mathrm{ms})$ and long (300to 500-ms) CFOAs, (ANOVA of $\arcsin p(C P, C S D)$, CFOA $\times$ $[\mathrm{FV}$, or OIa] $\times$ SOA interaction, $F(3,36)=2.45, p<.05$ ).

Presumably, Posner and Cohen's (1984) inhibition effect on the cued location is the cause for the reduced facilitation with 300- and 500-ms CFOAs. Inhibition cannot be completely suppressed by voluntary orienting when this location is repeatedly flashed (peripheral cue plus flash) and with 300to $500-\mathrm{ms}$ intervals between cue and flash onset (see also Maylor \& Hockey, 1987). Posner and Cohen's inhibition causes a second flash at the cued location to fail to reactivate fully the fast-acting (100-ms SOAs), reflexive mechanism after activation by a peripheral cue, and this is associated with a reduced strength of initial, passive inhibition for uncued locations. 


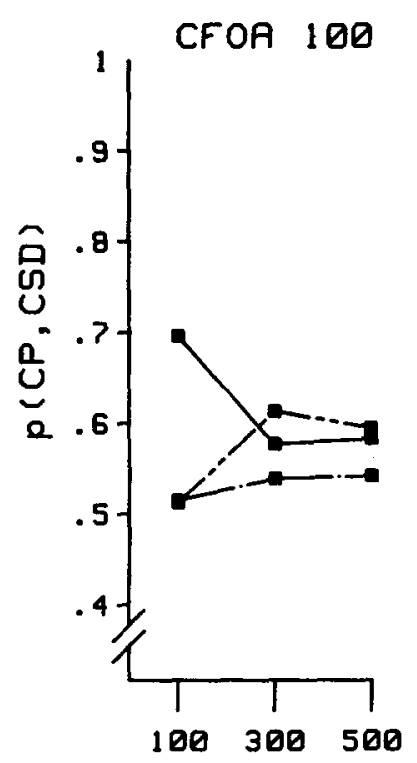

CFOA 300

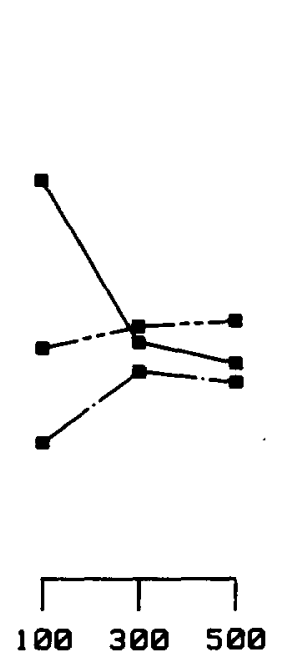

SOA (msec)
CFOA 500
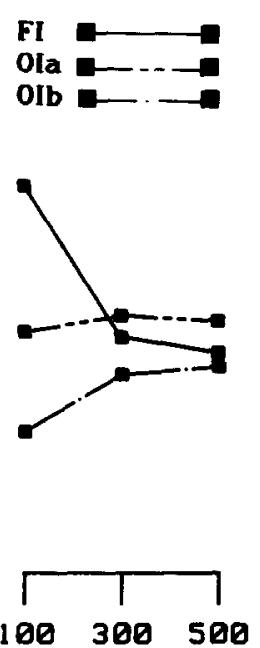

Figure 8. The probability of correct position-correct same-different responses, $p(C P, C S D)$, as a function of cue-flash onset asynchrony (CFOA) and flash-target stimulus onset asynchrony (SOA) plotted separately for target at other than flashed location, invalid (OI) trials with cue-compatible (a) and cue-incompatible (b) flashes and for target at flashed location, invalid (FI) trials.

\section{General Discussion}

The results of our study are consistent with the idea that spatial orienting can be achieved by two separate mechanisms, both addressing the same limited-capacity attention system. The reflexive mechanism engages attention automatically (Jonides, 1981). It cannot be prevented from producing an orienting response given its proper trigger stimulus (e.g., an abrupt change in light energy in extrafoveal vision), and once activated, it proceeds in an autonomous fashion; that is, it is characterized by strong resistance of response to competing stimuli. In contrast, the voluntary mechanism is affected by various processes. At least two kinds of processes can interfere with voluntary orienting: resource demands arising from competing cognitive tasks (Jonides, 1981; Posner, Cohen, Choate, Hockey, \& Maylor, 1984); and, most important in the context of our study, interference caused by the activation of the reflexive orienting mechanism (Experiment 2 and 3).

Two important questions concern whether activation of the reflexive orienting mechanism interferes with voluntary orienting only if the external trigger stimulus is incompatible with the cue (i.e., flash at uncued location) and whether activation of the reflexive orienting mechanism completely interrupts the voluntary mechanism or whether both mechanisms can be active at the same time, adding to or subtracting from each other (mixture) depending on whether they are compatible or not.

The first question is difficult to answer from our data. However, it seems a more plausible and parsimonious as- sumption that reflexive-to-voluntary interference always occurs when the reflexive mechanism is activated, whether its trigger stimulus is cue-compatible or not. This could provide an explanation for the finding (Experiment 3) that not only fast-acting reflexive orienting but also delayed-acting voluntary orienting may be affected by Posner and Cohen's (1984) inhibition effect if the likely location is flashed repeatedly (cue-flash-target sequence). According to the two-mechanism model, reflexive orienting to the flash at the cued location (100-ms SOA with 300- and 500-ms CFOAs) is reduced because of Posner and Cohen's inhibition; and voluntary orienting is reduced because reflexive-to-voluntary interference (300- and 500-ms SOAs with 300- and 500-ms CFOAs) may build up with repeated stimuli attempting to activate the reflexive orienting mechanism.

With regard to the second question, our data provide evidence against absolute interruption and for a mixture of the effects of reflexive and voluntary orienting. For instance, in Experiment 2 (central cuing involving the voluntary mechanism), facilitation for flashed locations was more marked when peripheral flash and central cue were compatible (facilitation for flashed cued locations) than when they were incompatible (facilitation for flashed uncued locations). Furthermore, inhibitory effects of cue-incompatible flashes on the other than flashed locations were less marked for likely (cued) than for unlikely (uncued) locations. That is, voluntary orienting, on the basis of a spatial set (cue validity), appears able to modify the effect of the reflexive mechanism: to attenuate it when cue and flash are incompatible, and to enhance it 


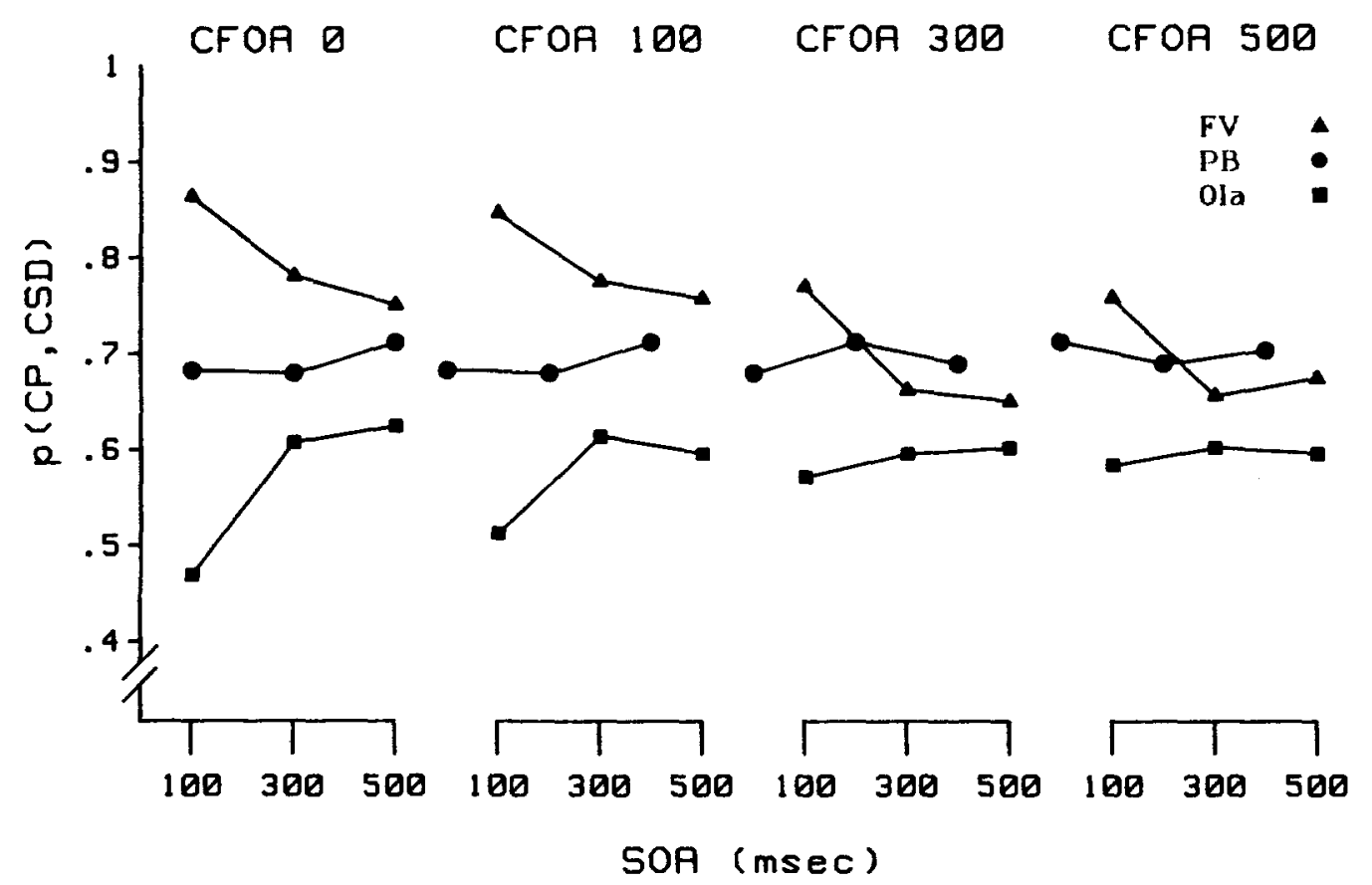

Figure 9. Cue-compatible flashes: The probability of correct position-correct same-different responses, $p(C P, C S D)$, as a function of cue-flash onset asynchrony (CFOA) and flash-target stimulus onset asynchrony (SOA) plotted separately for target at flashed location, valid (FV) trials, for peripheral cue only, baseline. (PB) trials, and for target at other than flashed location, invalid, flash at cued location (Ola) trials.

when they are compatible. This pattern is consistent with the idea that the reflexive and the voluntary mechanism can be active simultaneously.

The finding that the size of the effect of flashes depends on their cue compatibility (and on cue validity; see Jonides, 1980) seems inconsistent with an automaticity assumption that conceives of automaticity in absolute terms. However, the two-mechanism model resolves this problem: Reflexive orienting proceeds automatically given its trigger stimulus, whereas voluntary orienting is controlled, that is, guided by the strength of spatial expectancies that varies as a function of cue validity. Because reflexive-to-voluntary interference is not complete, overt performance depends on the combined action of both mechanisms. If the voluntary mechanism pulls in the same direction as the reflexive mechanism, their effects add to one another. If it pulls in a different direction, they subtract from one another (i.e., attention may be divided between different locations; see Eriksen \& Yeh, 1985). ${ }^{9}$ This is not to propose that the voluntary mechanism directly modifies the reflexive mechanism; rather, modification occurs indirectly through the strength of the effect of the voluntary mechanism on the limited-capacity attention system.

This idea has consequences for the interpretation of Experiment 1 , in particular the finding that peripheral cues produce stronger priming effects shortly after their onset than do peripheral and central cues at longer cue-target SOAs (see also Experiment 2, in particular the advantage for FV trials over CV trials, 100-ms after flash onset). Müller and Findlay (in press) interpreted this pattern as evidence for a powerful but transitory reflexive mechanism that is then replaced by a less effective but more persistent voluntary mechanism. However, although still maintaining that reflexive orienting comes into and goes out of play faster than voluntary orienting, the mixture account suggests that the greater priming effect with peripheral cues at short SOAs is caused by early voluntary orienting adding to the effect of reflexive orienting. Restated, the reflexive orienting mechanism on its own might not produce stronger cuing effects than voluntary orienting.

Yantis and Jonides (1984; Jonides \& Yantis, 1988) have demonstrated that reflexive orienting is selectively triggered by abrupt changes in light energy. Such changes are imperative, capturing attention possibly without effort, intention, and awareness. Yantis and Jonides linked this effect of direct peripheral cues with the special status of abrupt onsets in the transient visual system (e.g., Kulikowski \& Tolhust, 1973; Todd \& Van Gelder, 1979). In responding to abrupt changes in the periphery, this mechanism can be regarded as "part of an 'early warning system' that orients an organism and directs its attention to locations in visual space that ... contain novel pattern information" (Breitmeyer \& Ganz, 1976, p. 31).

Another important part of this system is the guidance of reflexive saccadic eye movements. The differential trade-off functions in Experiment 3 indicate that reflexive, covert ori-

\footnotetext{
${ }^{9}$ Eriksen and Yeh's (1985) trade-off between a primary and a secondary cued location is consistent with this account because in their displays the most likely position was directly cued by a bar marker (peripheral cuing) and the second most likely losation was always diagonally opposite to the cued positions (symbolic central cuing).
} 
enting fades within 100-300 ms after flash onset, that is, at a time characteristic for the latencies of overt saccadic eye movements. This suggests, together with other more direct evidence (e.g., Fischer \& Breitmeyer, 1987; Posner, Crippin, Cohen, \& Rafal, 1986), that reflexive shifts of attention precede saccadic changes of ocular fixation.

There has been some controversy concerning the nature of the relationship between saccadic eye movements and spatial orienting, that is, whether it is a necessary or a merely functional relationship (see Posner, 1980, for a review). The emerging view seems to be that reflexive orienting is intrinsically linked with saccade guidance but that voluntary orienting is not because voluntary shifts of attention and saccadic eye movements can be decoupled by experimental manipulations (Klein, 1980; Klein \& Hansen, 1987; but see Shepherd, Findlay, \& Hockey, 1986).

If there is an intrinsic relationship between reflexive orienting and saccadic eye movements, one plausible neural substrate of their link is the superior colliculus with its visual cells in the superficial layers and its oculomotor cells in the intermediate layers (Mohler \& Wurtz, 1976; Wurtz \& Mohler, 1976). Superficial layer cells show both a visual enhancement effect (Wurtz \& Mohler, 1976) and a remote inhibition effect (Richmond \& Wurtz, 1978; Rizzolati, Camarda, Grupp, \& Pisa, 1974). Surgical lesions of the superior colliculus reduce the distraction to a central visual discrimination task caused by peripheral flashes (Milner, Foreman, \& Goodale, 1978). All these properties are consistent with the reflexive mode of orienting. This is not to state that priming by peripheral cues is completely (or even mainly) explicable at the level of the superior colliculus; rather, other centers, such as the thalamic reticular complex (Crick, 1984), are presumably involved.

The neural basis of the voluntary mode of orienting is more elusive, but the posterior parietal cortex may be a plausible site (Lynch, 1980; Mountcastle, 1978). In particular, cells in area 7 of the posterior parietal cortex show enhancement of visual responses, which is independent of the particular task required: eye or hand movement (Bushnell, Goldberg, \& Robinson, 1981). This suggests involvement of cells in the posterior parietal cortex in the higher-level control system of spatial orienting.

Posner et al. (1985) proposed that the higher-level system provides "the basis for orienting ... such as in terms of voluntary commands to move attention" to particular spatial locations and the lower centers exercise "the more primitive controls such as over the rate of movement" (p. 222). However, although our findings support the distinction between higher- and lower-level mechanisms of spatial orienting, they also question whether they are best described in terms of a coupled hierarchy of a subordinate executive mechanism guided by a superordinate command mechanism. Rather, the reflexive (i.e., more primitive) orienting mechanism seems to operate as an autonomous module that can be modified but not suppressed by higher-level control.

\section{References}

Breitmeyer, B. G., \& Ganz, L. (1976). Implications of sustained and transient channels for theories of visual pattern masking, saccadic suppression, and information processing. Psychological Review, 83, $1-36$.
Bushnell, M. C., Goldberg, M. E., \& Robinson, D. L. (1981). Behavioral enhancement of visual response in monkey cerebral cortex: $I$. Modulation in posterior parietal cortex related to selective attention. Journal of Neurophysiology, 46, 755-772.

Colegate, R. L., Hoffman, J. E., \& Eriksen, C. W. (1973). Selective encoding from multielement visual displays. Perception \& Psycho physics, 14, 217-224.

Crick, F. (1984). Function of the thalamic reticular complex: The search light hypothesis. Proceedings of the National Academy of Sciences of the United States of America, 81, 4586-4590.

Duncan, J. (1980a). The demonstration of capacity limitation. Cognitive Psychology, 12, 75-96.

Duncan, J. (1980b). The locus of interference in the perception of simultaneous stimuli. Psychological Review, 87, 272-300.

Eriksen, C. W., \& Colegate, R. L. (1971). Selective attention and serial processing in briefly presented visual displays. Perception \& Psychophysics, 10, 321-326.

Eriksen, C. W., \& Hoffman, J. E. (1972). Some characteristics of selective attention in visual perception determined by vocal reaction times. Perception \& Psychophysics, 11, 169-171.

Eriksen, C. W., \& Yeh, Y.-Y. (1985). Allocation of attention in the visual field. Journal of Experimental Psychology: Human Perception and Performance, 11, 583-597.

Findlay, J. M. (1974). A simple apparatus for recording microsaccades during fixation. Quarterly Journal of Experimental Psychology, 26, 167-170.

Findlay, J. M. (1978). Estimates on probability functions: A more virulent PEST. Perception \& Psychophysics, 23, 181-185.

Findlay, J. M. (1981). Spatial and temporal factors in the predictive generation of saccadic eye movements. Vision Research, 21, 347354.

Fischer, B., \& Breitmeyer, B. (1987). Mechanisms of visual attention revealed by saccadic eye movements. Neuropsychologia, 25, 7383.

Jonides, J. (1980). Towards a model of the mind's eye's movement. Canadian Journal of Psychology, 34, 103-112.

Jonides, J. (1981). Voluntary versus automatic control over the mind's eye. In J. Long \& A. Baddeley (Eds.), Attention and performance $I X$ (pp. 187-203). Hillsdale, NJ: Erlbaum.

Jonides, J., \& Yantis, S. (1988). Uniqueness of abrupt visual onset in capturing attention. Perception \& Psychophysics, 43, 346-354.

Klein, R. (1980). Does oculomotor readiness mediate cognitive control of visual attention? In R. S. Nickerson (Ed.), Attention and performance VIII (pp. 259-276). Hillsdale, NJ: Erlbaum.

Klein, R., \& Hansen, E. (1987). Spotlight failure in covert visual orienting. Bulletin of the Psychonomic Society, 25, 447-450.

Kowler, E., \& Steinman, R. M. (1979a). The effect of expectations on slow oculomotor control: I. Periodic target steps. Vision Research, 19, 612-632.

Kowler, E., \& Steinman, R. M. (1979b). The effect of expectations on slow oculomotor control: II. Single target displacements. Vision Research, 19, 633-646.

Kulikowski, J. J., \& Tolhurst, D. J. (1973). Psychological evidence for sustained and transient detectors in human vision. Journal of Physiology, 232, 149-162.

LaBerge, D. (1981). Automatic information processing: A review. In J. Long \& A. Baddeley (Eds.), Attention and performance IX (pp. 173-186). Hillsdale, NJ: Erlbaum.

Lynch, J. C. (1980). The functional organization of posterior parietal association cortex. Behavioral and Brain Sciences, 3, 485-534.

McLean, J. P., \& Shulman, G. L. (1978). On the construction and maintenance of expectancies. Quarterly Journal of Experimental Psychology, 30, 441-454.

Maylor, E. A. (1985). Facilitatory and inhibitory components of orienting in visual space. In M. I. Posner \& O. S. M. Marin (Eds.), Attention and performance $X I$ (pp. 189-204). Hillsdale, NJ: Erl- 
baum.

Maylor, E. A., \& Hockey, R. (1987). Effects of repetition on the facilitatory and inhibitory components of orienting in visual space. Neuropsychologia, 25, 41-54.

Milner, A. D., Foreman, N. P., \& Goodale, M. A. (1978). Go-left goright discrimination performance and distractability following lesions of prefrontal cortex or superior colliculus in stumptail macaques. Neuropsychologia, 16, 381-390.

Mohler, C. W., \& Wurtz, R. (1976). Organization of monkey superior colliculus: Intermediate layer cells discharging before eye movements. Journal of Neurophysiology, 39, 722-744.

Mountcastle, V. B. (1978). Brain mechanisms for directed attention. Journal of the Royal Society of Medicine, 71, 14-28.

Müller, H. J. (1984, January). The effect of selective spatial attention on peripheral discrimination thresholds. Paper presented at the meeting of the Experimental Psychology Society, London.

Müller, H. J., \& Findlay, J. M. (1987). Sensitivity and criterion effects in the spatial cuing of visual attention. Perception \& Psychophysics, 42, 383-399.

Müller, H. J., \& Findlay, J. M. (in press). The effect of visual attention on peripheral discrimination thresholds in single and multiple element displays. Acta Psychologica.

Müller, H. J., \& Rabbitt, P. M. A. (1988). Spatial cuing and the relation between the accuracy of "where" and "what" decisions in visual search. Manuscript submitted for publication.

Navon, D. (1984). Resources-A theoretical soup stone? Psychological Review, 91, 216-234.

Posner, M. I. (1980). Orienting of attention. Quarterly Journal of Experimental Psychology, 32, 3-25.

Posner, M. I., \& Cohen, Y. (1984). Components of visual orienting. In H. Bouma \& D. G. Bouwhuis (Eds.), Attention and performance $X$ (pp. 531-555). Hillsdale, NJ: Erlbaum.

Posner, M. I., Cohen, Y., Choate, L. S., Hockey, R., \& Maylor, E. (1984). Sustained concentration: Passive filtering or active orienting? In S. Kornblum \& J. Requin (Eds.), Preparatory states and processes (pp. 49-65). Hillsdale, NJ: Erlbaum.

Posner, M. I., Crippin, P. J., Cohen, A., \& Rafal, R. (1986, November). Speed of covert orienting of attention and express saccades 1 . Paper presented at the meeting of the Psychonomic Society, New Orleans.

Posner, M. I., Rafal, R. D., Choate, L. S., \& Vaughan, J. (1985).
Inhibition of return: Neural basis and function. Cognitive Neuropsychology, 2, 211-228.

Posner, M. I., Snyder, C. R. R., \& Davidson, B. J. (1980). Attention and the detection of signals. Journal of Experimental Psychology: General, 109, 160-174.

Richmond, B. J., \& Wurtz, R. H. (1978). Visual masking by remote stimuli in monkey superior colliculus. Neuroscience Abstracts, 4 , 642.

Rizzolati, G., Camarda, R., Grupp, L. A., \& Pisa, M. (1974). Inhibitory effect of remote visual stimuli on visual responses of cat superior colliculus: Spatial and temporal factors. Journal of Neurophysiology, 37, 1262-1275.

Schneider, W., \& Shiffrin, R. M. (1977). Controlled and automatic human information processing: I. Detection, search, and attention. Psychological Review, 84, 1-66.

Shepherd, M. (1984). EMDISP: A visual display system with digital and analogue sampling. Behavior Research Methods, Instruments, and Computers, 16, 297-302.

Shepherd, M., Findlay, J. M., \& Hockey, R. J. (1986). The relationship between eye movements and spatial attention. Quarterly Journal of Experimental Psychology, 38, 475-491.

Shiffrin, R. M., \& Schneider, W. (1977). Controlled and automatic human information processing: II. Perceptual learning, automatic attending, and a general theory. Psychological Review, 84, 127 190.

Todd, J. T., \& Van Gelder, P. (1979). Implications of a transientsustained dichotomy for the measurement of human performance. Journal of Experimental Psychology: Human Perception and Performance, 5, 625-638.

Winer, B. J. (1971). Statistical principles in experimental design (2nd ed.). New York: McGraw-Hill.

Wurtz, R., \& Mohler, C. W. (1976). Organization of monkey superior colliculus: Enhanced visual response of superficial layer cells. Journal of Neurophysiology, 39, 745-765.

Yantis, S., \& Jonides, J. (1984). Abrupt visual onsets and selective attention: Evidence from visual search. Journal of Experimental Psychology: Human Perception and Performance, 10, 601-621.

Received February 14, 1986 Revision received June 15, 1988 Accepted June 17, 1988 